\title{
Mitigating the Effects of the Space Radiation Environment: A Novel Approach of Using Graded-Z Materials
}

\author{
William Atwell ${ }^{1}$ \\ Boeing Research \& Technology (BR\&T), Houston, TX 77058 \\ Kristina Rojdev ${ }^{2}$ \\ NASA Johnson Space Center, Houston, TX 77058 \\ Sukesh Aghara ${ }^{3}$ and Sirikul Sriprisan ${ }^{4}$ \\ University of Massachusetts Lowell, Lowell, MA 01854
}

\begin{abstract}
In this paper we present a novel space radiation shielding approach using various material lay-ups, called "Graded-Z" shielding, which could optimize cost, weight, and safety while mitigating the radiation exposures from the trapped radiation and solar proton environments, as well as the galactic cosmic radiation (GCR) environment, to humans and electronics. In addition, a validation and verification (V\&V) was performed using two different high energy particle transport/dose codes (MCNPX \& HZETRN). Inherently, we know that materials having high-hydrogen content are very good space radiation shielding materials. Graded-Z material lay-ups are very good trapped electron mitigators for medium earth orbit (MEO) and geostationary earth orbit (GEO). In addition, secondary particles, namely neutrons, are produced as the primary particles penetrate a spacecraft, which can have deleterious effects to both humans and electronics. The use of "dopants," such as beryllium, boron, and lithium, impregnated in other shielding materials provides a means of absorbing the secondary neutrons. Several examples of optimized Graded-Z shielding layups that include the use of composite materials are presented and discussed in detail. This parametric shielding study is an extension of some earlier pioneering work we (William Atwell and Kristina Rojdev) performed in $2004^{1}$ and $2009^{2}$.
\end{abstract}

\section{Nomenclature}

GCR = galactic cosmic radiation

GEO = geostationary earth orbit

HZETRN = High Z and Energy Transport code

MCNPX $=$ Monte Carlo Nuclear Particle transport computer code

$\mathrm{MEO}=$ medium earth orbit

$\mathrm{V} \& \mathrm{~V}=$ validation \& verification

\section{Introduction}

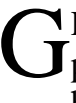

RADED-Z shielding is a laminate of several materials with different $\mathrm{Z}$ values (atomic numbers) designed to protect against ionizing radiation. Compared to single-material shielding, the same mass of Graded-Z shielding has been shown to reduce electron penetration over $60 \% .{ }^{3}$ Graded-Z shielding is commonly used in satellitebased particle detectors and offers several benefits: protection from radiation damage, reduction of background noise for detectors, and lower mass compared to single-material shielding. Designs vary, but typically involve a gradient

\footnotetext{
${ }^{1}$ Technical Fellow, Boeing Research \& Technology, 13100 Space Center Blvd./HB 2-30, AIAA Associate Fellow.

2 Engineer, Systems Architecture and Integration Office, 2101 NASA Parkway, MC: EA-361, AIAA Student Member.

${ }^{3}$ Associate Professor, Chemical Engineering (Nuclear), 1 University Ave., Perry Hall - 318.

${ }^{4}$ Research Associate, Chemical Engineering (Nuclear), 1 University Ave., Perry Hall - 318.
} 
from high-Z (usually tantalum [Ta]) through successively lower-Z elements such as tin, steel, and copper, usually ending with aluminum. Sometimes even lighter materials such as polypropylene and boron carbide are used. ${ }^{4,5}$ In a typical Graded-Z shield, the high-Z layer effectively scatters protons and electrons. It also absorbs gamma rays, which produces X-ray fluorescence. Each subsequent layer absorbs the X-ray fluorescence of the previous material, eventually reducing the energy to a suitable level. Each decrease in energy produces bremsstrahlung and Auger electrons, which are below the detector's energy threshold. Some designs also include an outer layer of aluminum, which may simply be the outer structure of a spacecraft or satellite. Fan, et al. ${ }^{6}$ reports "Shielding for space microelectronics needs to provide an acceptable dose rate with minimum shield mass. The analysis presented here shows that the best approach is, in general, to use a graded- $Z$ shield, with a high-Z layer sandwiched between two low-Z materials. A Graded-Z shield is shown to reduce the electron dose rate by more than sixty percent over a single-material shield of the same areal density. For protons, the optimal shield would consist of a single, low- $Z$ material layer. However, it is shown that a Graded-Z shield is nearly as effective as a single-material shield, as long as a low-Z layer is located adjacent to the microelectronics. A specific shield design depends upon the details of the radiation environment, system model, design margins/levels, compatibility of shield materials, etc.”

In this paper we investigate several novel Graded- $Z$ materials that are compared with baseline materials: aluminum (Al), high density polyethylene (HDPE), and water $\left(\mathrm{H}_{2} \mathrm{O}\right)$. In addition, we compare the results using two different computational computer codes, NASA HZETRN ${ }^{7}$ and MCNPX $^{8}$ to validate and verify our results.

\section{Study Assumptions}

\section{A. Graded-Z configurations}

The shielding configurations considered in this paper were two-layer shields with typical shielding thicknesses and very large thickness configurations. The two layer shielding configurations were Al-HDPE, Ta-HDPE, and WHDPE. The thicknesses of Al, Ta, and W were 150 mils, which correspond to 1.0287, 6.3237, and $7.3343 \mathrm{~g} / \mathrm{cm}^{2}$, respectively, and the HDPE thicknesses were 5 and $10 \mathrm{~g} / \mathrm{cm}^{2}$. The very large thickness configurations consisted of single layer $\mathrm{H}_{2} \mathrm{O}$ and two-layer $\mathrm{H}_{2} \mathrm{O}-\mathrm{SWX}$. SWX, specifically SWX-210, is a boron-doped hydrocarbon having a density of $1.19 \mathrm{~g} / \mathrm{cm}^{3}$. The water thicknesses ranged from $100-300 \mathrm{~g} / \mathrm{cm}^{2}$, and the SWX thicknesses were 10 and 20 $\mathrm{g} / \mathrm{cm}^{2}$.

\section{B. Environments}

For deep-space exploratory missions, the radiation sources are due to the naturally-occurring galactic cosmic radiation (GCR) and from solar particle events (SPEs). The radiation exposure analyses discussed later is based on the GCR environment during solar minimum (epoch 1977), solar maximum (epoch 1982), and the classic series of solar proton events that occurred 19-24 October 1989. The October 1989 SPEs were four events that were combined

using the Band fit. ${ }^{9,10}$ For all three environment cases, the energy spectrum was restricted to $2 \mathrm{GeV}$ due to limitations in the MCNPX software, as shown in the differential energy spectrum below (Figure 1). 


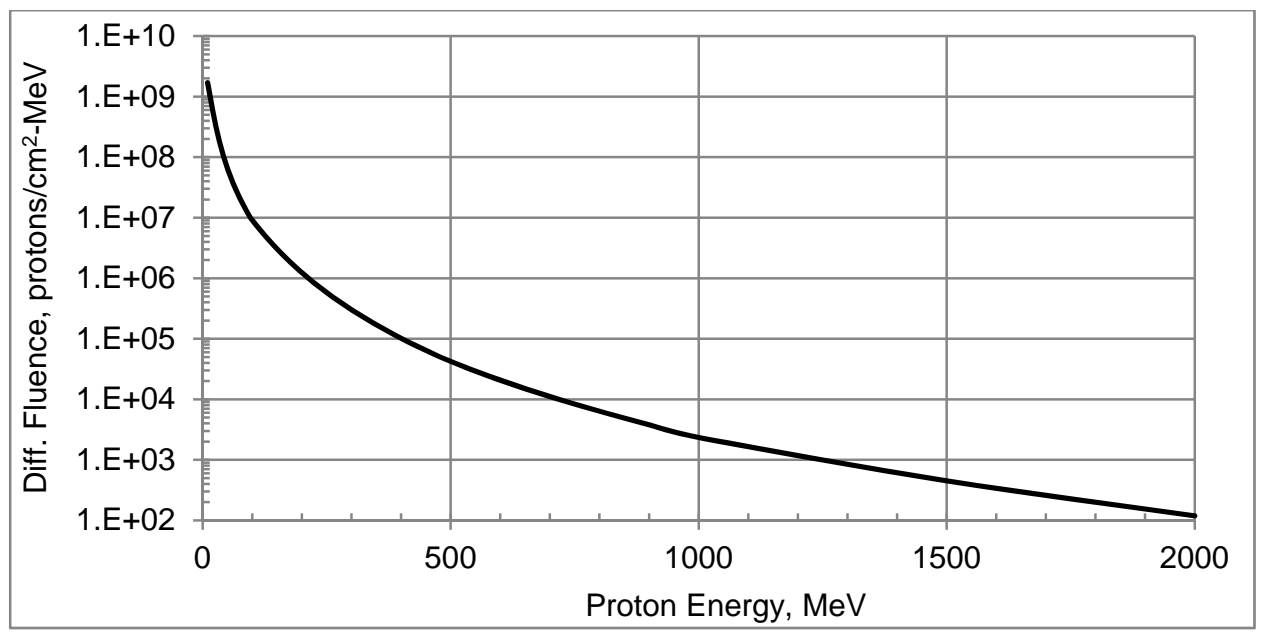

Figure 1. The Band fit differential energy spectrum for the 19-24 October 1989 SPEs.

\section{High Energy Particle Transport/Dose Codes}

Two high energy particle transport/dose codes were used in the computations, HZETRN ${ }^{7}$ and MCNPX ${ }^{8}$, and the results were compared as a check on the verification and validation of the absorbed doses.

The HZETRN code was develop at NASA Langley Research Center in 1995. The high-charge-and energy (HZE) transport computer program HZETRN was developed to address the problems of free-space radiation transport and shielding. The HZETRN program is intended specifically for the design engineer who is interested in obtaining fast and accurate dosimetric information for the design and construction of space modules and devices. The program is based on a one-dimensional space-marching formulation of the Boltzmann transport equation with a straight-ahead approximation. The effect of the long-range Coulomb force and electron interaction is treated as a continuous slowing-down process. Atomic (electronic) stopping power coefficients with energies above a few $\mathrm{MeV}$ are calculated by using Bethe`s theory including Bragg`s rule, Ziegler`s shell corrections, and effective charge. Nuclear absorption cross sections are obtained from fits to quantum calculations and total cross sections are obtained with a Ramsauer formalism. Nuclear fragmentation cross sections are calculated with a semi-empirical abrasion-ablation fragmentation model. The relation of the final computer code to the Boltzmann equation is discussed in the context of simplifying assumptions.

The MCNPX code was developed at Los Alamos National Laboratory (LANL) and is described at their website $^{11}$ as "MCNP is a general-purpose Monte Carlo N-Particle code that can be used for neutron, photon, electron, or coupled neutron/photon/electron transport. Specific areas of application include, but are not limited to, radiation protection and dosimetry, radiation shielding, radiography, medical physics, nuclear criticality safety, detector design and analysis, nuclear oil well logging, accelerator target design, fission and fusion reactor design, decontamination and decommissioning. The code treats an arbitrary three-dimensional configuration of materials in geometric cells bounded by first- and second-degree surfaces and fourth-degree elliptical tori. Point-wise crosssection data typically are used, although group-wise data also are available. For neutrons, all reactions given in a particular cross-section evaluation (such as ENDF/B-VI) are accounted for. Thermal neutrons are described by both the free gas and S(alpha,beta) models. For photons, the code accounts for incoherent and coherent scattering, the possibility of fluorescent emission after photoelectric absorption, absorption in pair production with local emission of annihilation radiation, and bremsstrahlung. A continuous-slowing-down model is used for electron transport that includes positrons, $\mathrm{x}$-rays, and bremsstrahlung but does not include external or self-induced fields. Important standard features that make MCNP very versatile and easy to use include a powerful general source, criticality source, and surface source; both geometry and output tally plotters; a rich collection of variance reduction techniques; a flexible tally structure; and an extensive collection of cross-section data. MCNP contains numerous flexible tallies: surface current \& flux, volume flux (track length), point or ring detectors, particle heating, fission heating, pulse height tally for energy or charge deposition, mesh tallies, and radiography tallies.” 


\section{Results}

\section{A. Two-layer Configurations}

There were three arrangements investigated for the two-layer configurations. The outer layer was either aluminum (Al), Tantalum (Ta), or Tungsten (W) and corresponded to 150 mils of thickness. The inner layers were high density polyethylene (HDPE) at 5 or $10 \mathrm{~g} / \mathrm{cm}^{2}$ thickness. These layers were compared with the same thicknesses of Al or HDPE.

The results for the Al-HDPE system (Figure 2) show that the single layer aluminum case has the worst dose, while the Graded-Z Al-HDPE and single layer HDPE systems are comparable in dose.

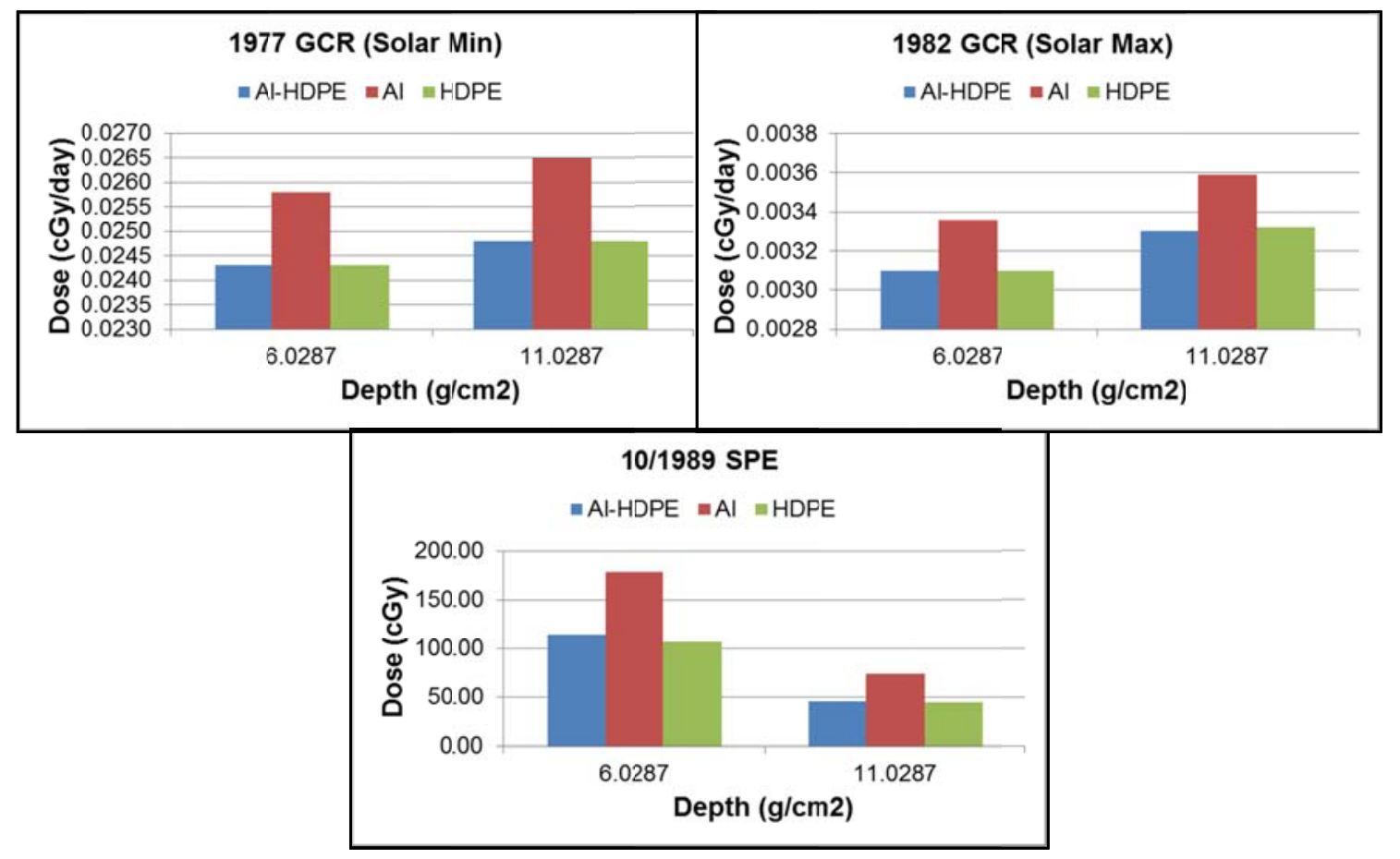

Figure 2. Al-HDPE two-layer system compared with Aluminum and HDPE at the same thicknesses.

The results of the Ta-HDPE system (Figure 3) show similar results to the Al-HDPE case. For the GCR environments, the Ta-HDPE system with $5 \mathrm{~g} / \mathrm{cm}^{2}$ of HDPE is slightly superior to the single layer of HDPE. However, the Ta-HDPE case with $10 \mathrm{~g} / \mathrm{cm}^{2}$ of HDPE is comparable to the single layer of HDPE. For the SPE environment, the reverse is true. The Ta-HDPE system is now comparable with the single layer of aluminum and the single layer of HDPE is superior for dose mitigation. 


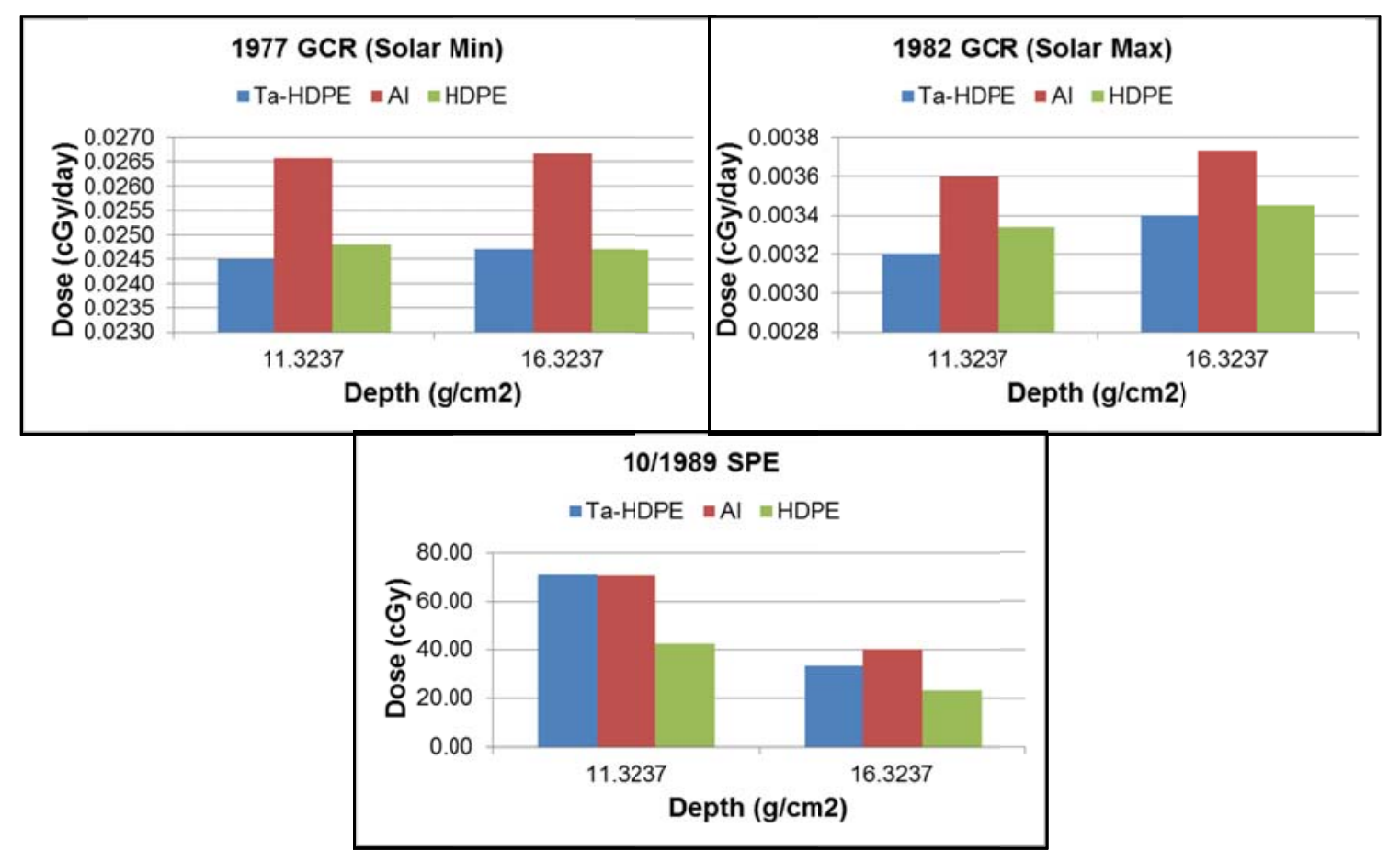

Figure 3. Ta-HDPE two-layer system compared with Aluminum and HDPE at the same thicknesses.

The results of the W-HDPE system (Figure 4) show similar results to the Ta-HDPE case. For the GCR environments, the W-HDPE system with $5 \mathrm{~g} / \mathrm{cm}^{2}$ of HDPE is slightly superior, but the W-HDPE system with 10 $\mathrm{g} / \mathrm{cm}^{2}$ of HDPE is more comparable to the single layer of HDPE. For the SPE environment, the W-HDPE system is more comparable to the single layer of aluminum, and the single layer of HDPE is the best radiation mitigator. 


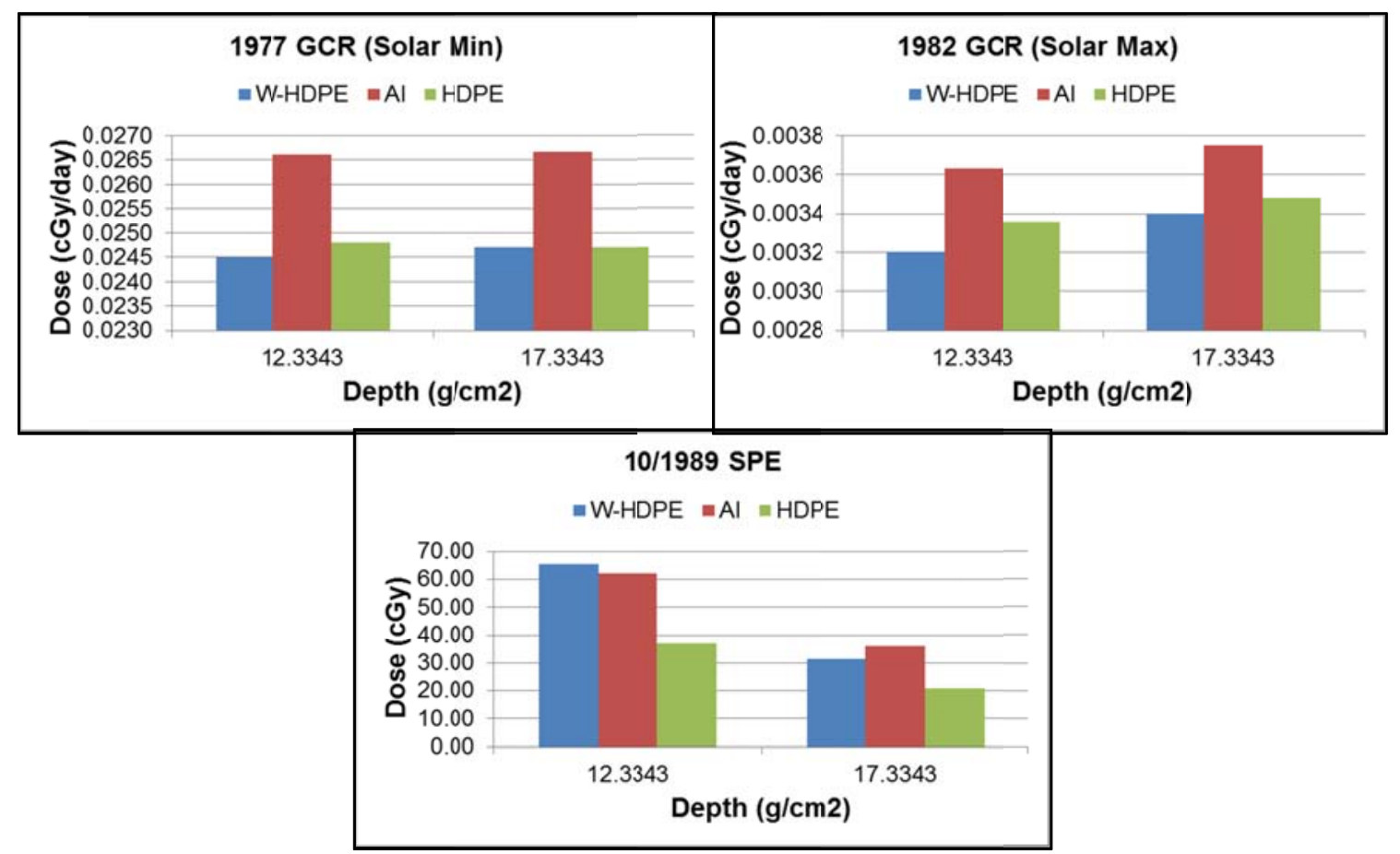

Figure 4. W-HDPE two-layer system compared with Aluminum and HDPE at the same thicknesses.

The two layer configurations discussed in this section are similar to historical spacecraft thicknesses for radiation shielding purposes. The next section will consider very large thicknesses of water and whether the larger thicknesses change these results.

\section{B. Very Large Thickness Configurations}

For the very large thickness configurations, there were six designs analyzed that consisted of combinations of water with a borated polyethylene known as SWX. The three thicknesses of water were 100,200 , and $300 \mathrm{~g} / \mathrm{cm}^{2}$ and the two thicknesses of SWX considered were 10 and $20 \mathrm{~g} / \mathrm{cm}^{2}$. For comparison, a single layer of water at the same overall thicknesses was also considered. The results (Figure 5) show that the $\mathrm{H}_{2} \mathrm{O}-\mathrm{SWX}$ systems are comparable to the single layer of $\mathrm{H}_{2} \mathrm{O}$ at the same thicknesses. 


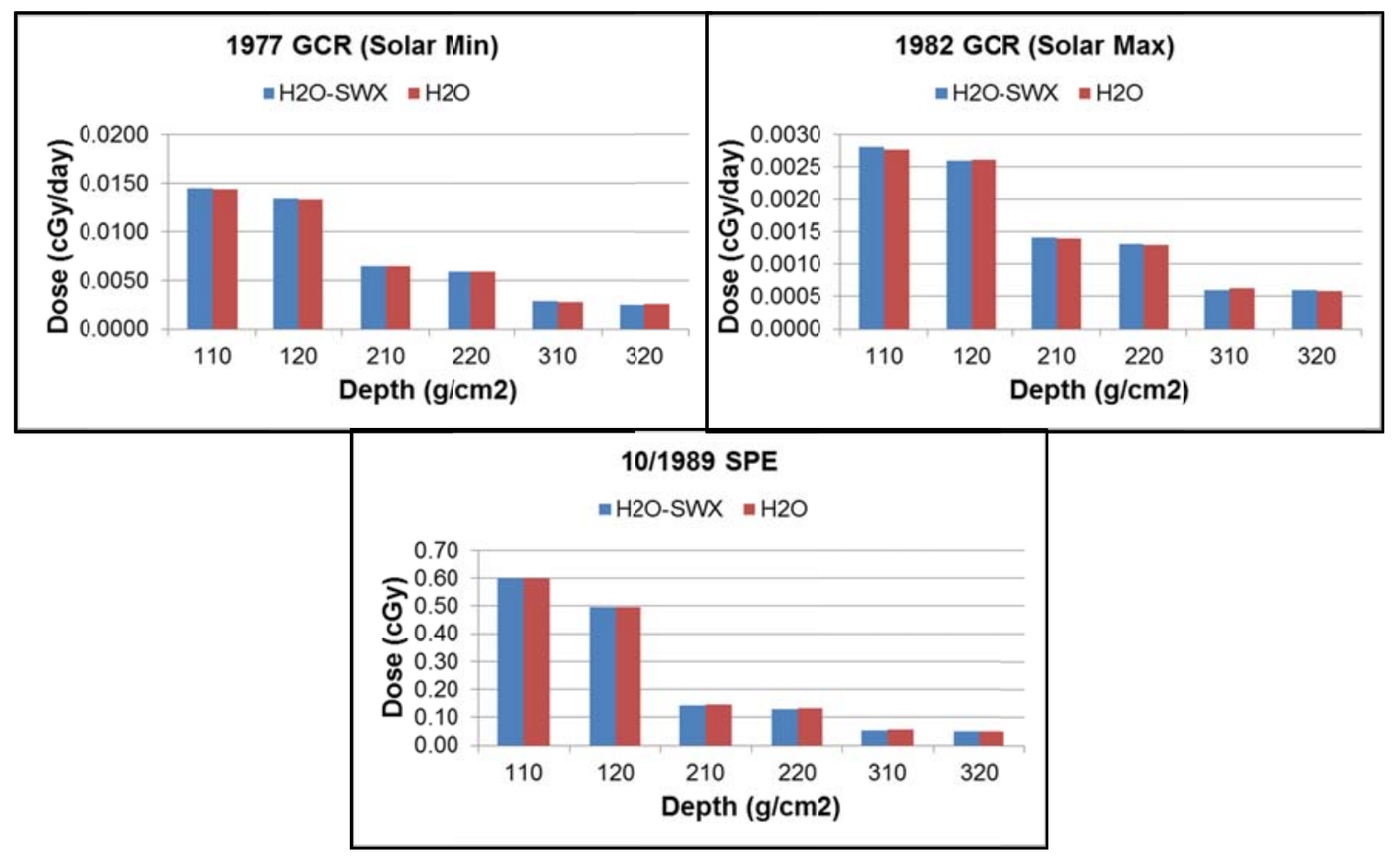

Figure 5. H2O-SWX very large thickness system compared with single layer of $\mathrm{H} 2 \mathrm{O}$ at the same thicknesses.

\section{Verification and Validation}

For the verification and validation, high energy proton particles produced by SPE events and the proton component of the GCR are transported through shield material and tissue equivalent geometry using the Monte Carlo nuclear particle transport computer code, MCNPX. The MCNPX simulations were performed for the twolayer configurations and one case of the very large thickness configuration, for each environment case.

The radiation source (GCR or SPE) spectra is modeled as an uniformly distributed planar source propagating from left to right along $\mathrm{z}$ axis toward a simple cylinder (of $15 \mathrm{~cm}$ radius). The input spectra source ranges from 0.01 $\mathrm{MeV}$ to $2 \mathrm{GeV}$. The radial surface of the cylinder is modeled as a reflecting boundary to simulate an infinite material in the radial direction and to accomplish a one dimensional simulation. This allows for comparisons between MCNPX and HZETRN results. The MCNPX simulations are performed with the following particles transported explicitly: neutrons, protons, photons, electrons and light particles including deuteron, triton, helium- 3 , and alphas. The photon-electron transport is not present in the HZETRN version used in this study.

The MCNPX code uses the mix-match algorithm that utilizes nuclear data tables to transport particles at certain energy ranges, and intranuclear cascade (INC) physics models to handle interactions at high energy regions. ${ }^{12}$ The combinations of flux tally (F4), energy depositions per track length tally (F6), and cylindrical mesh tally were used to generate results. The F4 tallies were used to evaluate energy spectra at a transition point between shielding, at tissue phantom surfaces, and at various phantom depths. The F6 tally is used to calculate absorbed dose, averaged over a cell volume, resulting in dose (Gy/source particle/s).

All calculations presented in this study are performed with MCNPX version 2.7.0. All simulations were performed at a total of 2 million histories. The calculated results were set to meet a minimum of $10 \%$ or less statistical uncertainty. The corresponding error bars were plotted in the curves, but in most cases, they were not easy to see because of its trivial values.

Figure 6 shows the plot of neutron flux (secondary neutrons produced, resulting from the primary protons of the SPE) for the five different shielding cases. It is evident that the neutron fluxes (10-150 MeV) in the water shields are substantially lower as compared to the Graded-Z shield cases. Figure $\mathbf{7}$ and Figure 8 show the flux plots for the GCR (solar minimum and maximum) cases, respectively. Again notice the neutron fluxes for the water shield to be lower compared to the Graded-Z shield cases. Additionally, we notice (more pronounced) that the SWX layers in combination with water produce a higher thermal neutron flux as compared to the water case. This may be due to a 
higher primary proton interaction with the shield material, resulting in a higher secondary neutron production. When these results are viewed in context with the dose results, it shows that the does is lower for the SWX cases when compared to just the water case. This suggests that the more damaging protons are removed by the SWX, providing additional shielding.

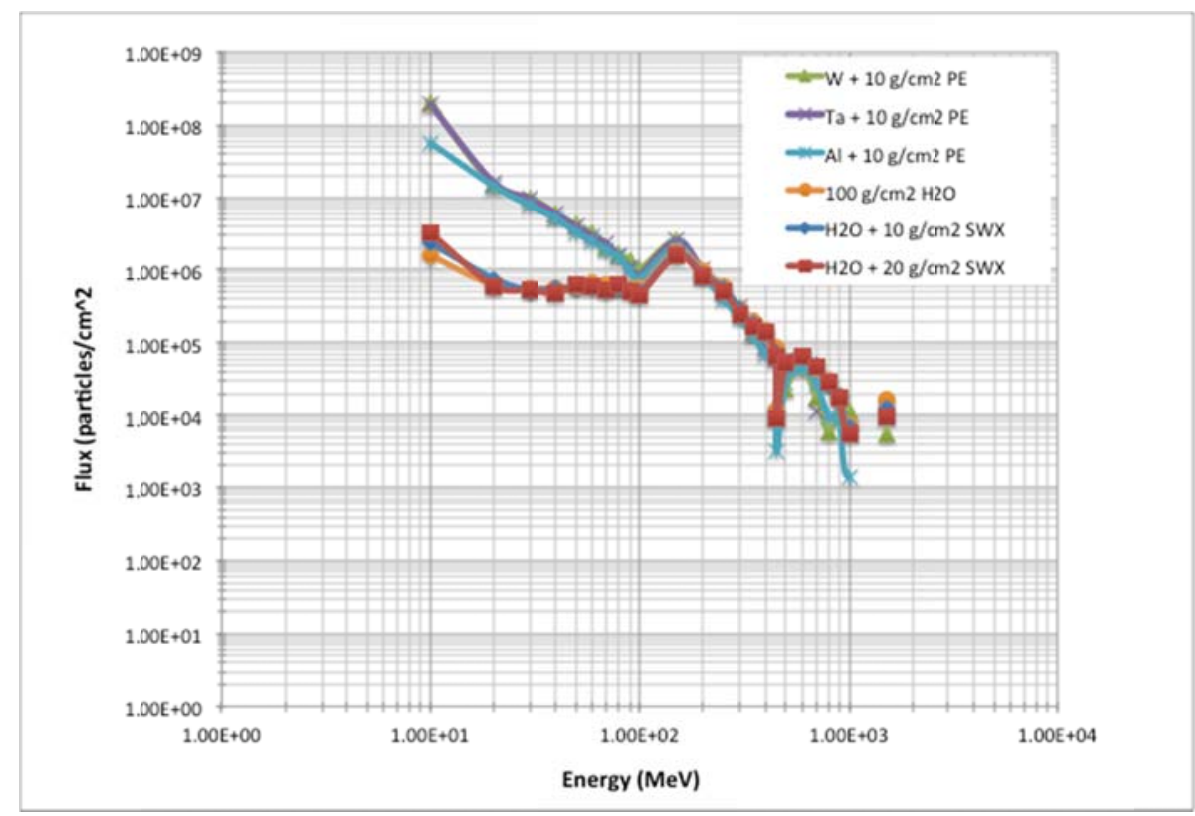

Figure 6. October 1989 SPE Band fit neutron flux.

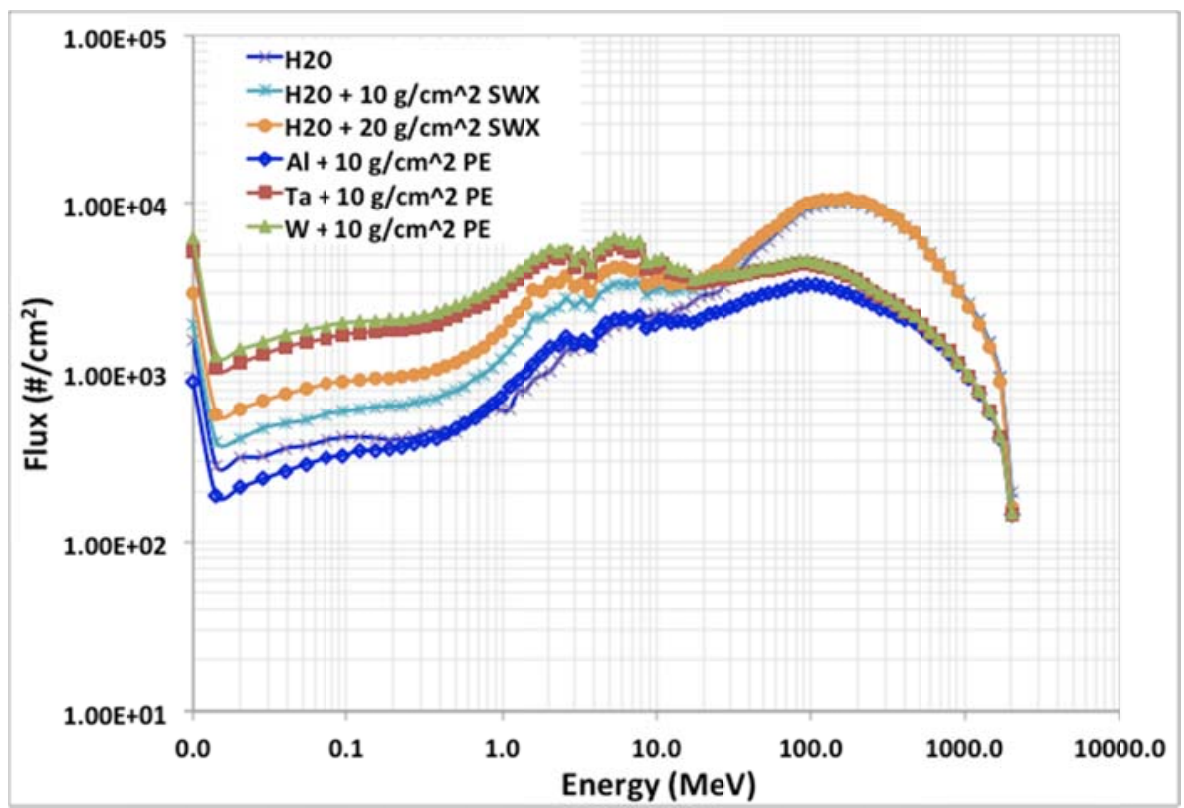

Figure 7. 1977 GCR (solar minimum) neutron flux 


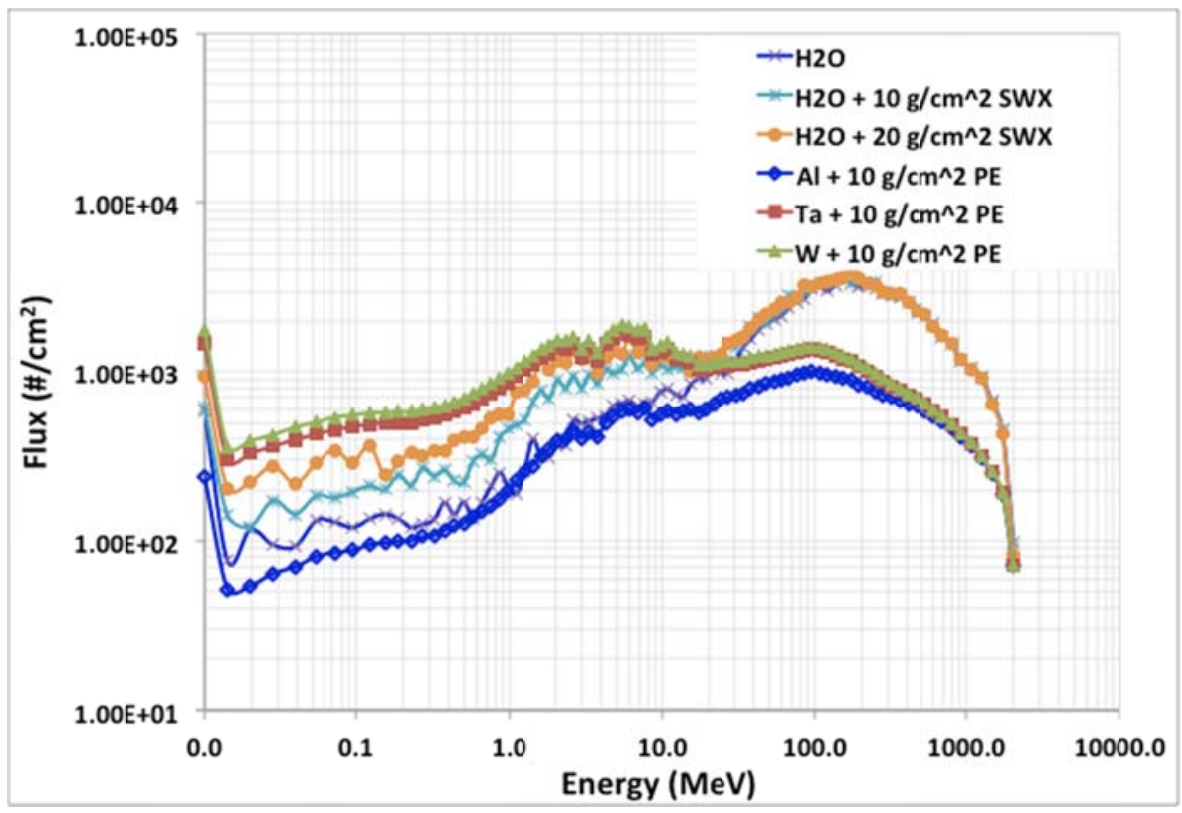

Figure 8. 1982 GCR (solar maximum) neutron flux.

Table 1 shows the summary of MCNPX dose results for the SPE. Clearly the dose values show a consistent trend to the HZETRN results. Overall, the MCNPX dose values are $15 \%$ to $30 \%$ higher than the HZETRN dose values. These results are consistent with previously published work. ${ }^{12,13}$ The absence of EM transport in HZETRN is one of the major reasons for these differences. Also the 2005 version of HZETRN does not include the upgrades to the neutron and light ion transport now available in the 2010 release. ${ }^{14}$ This also contributes to the differences between the results. When the ratios of the relative thicknesses of the shields are compared, the difference between MCNPX and HZETRN is much smaller (5-10\%). This is stated as the D ratio. This difference may be due to the differences in the transport calculation algorithms and in the cross-sections used by the two codes. The differences are higher for the 1982 GCR solar maximum results (Table 3), approximately 50\% higher. Further investigation is necessary to understand why the difference is much greater than what we observe for the SPE and the 1977 GCR solar minimum results. 
Table 1. Dose values for October 1989 SPE Band fit.

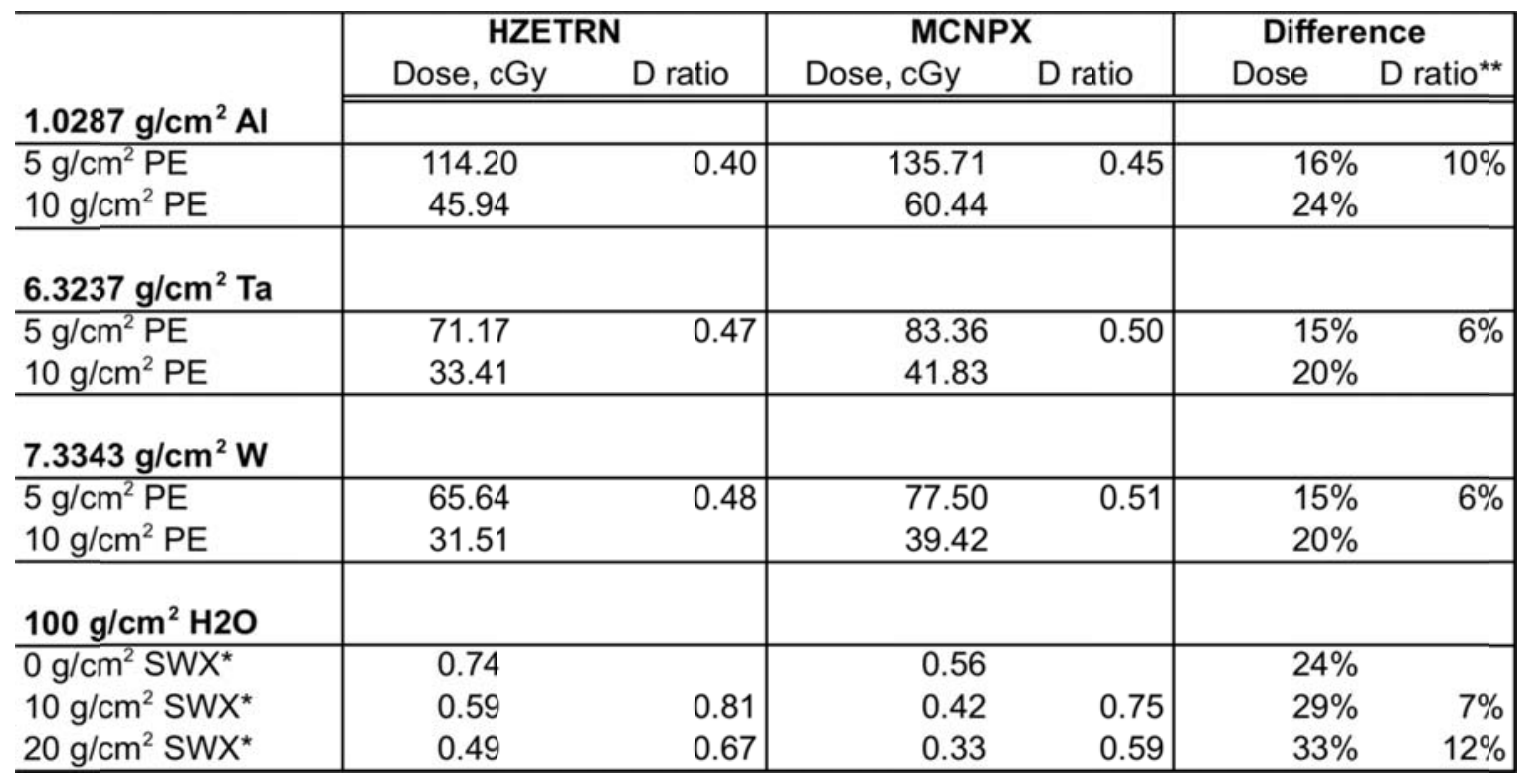

*here the HZETRN dose is higher than MCNPX

**the D ratio is the ratio of dose values from $10 \mathrm{~g} / \mathrm{cm} 2$ shield to the $5 \mathrm{~g} / \mathrm{cm} 2$

Table 2. Dose values for 1977 GCR (solar minimum).

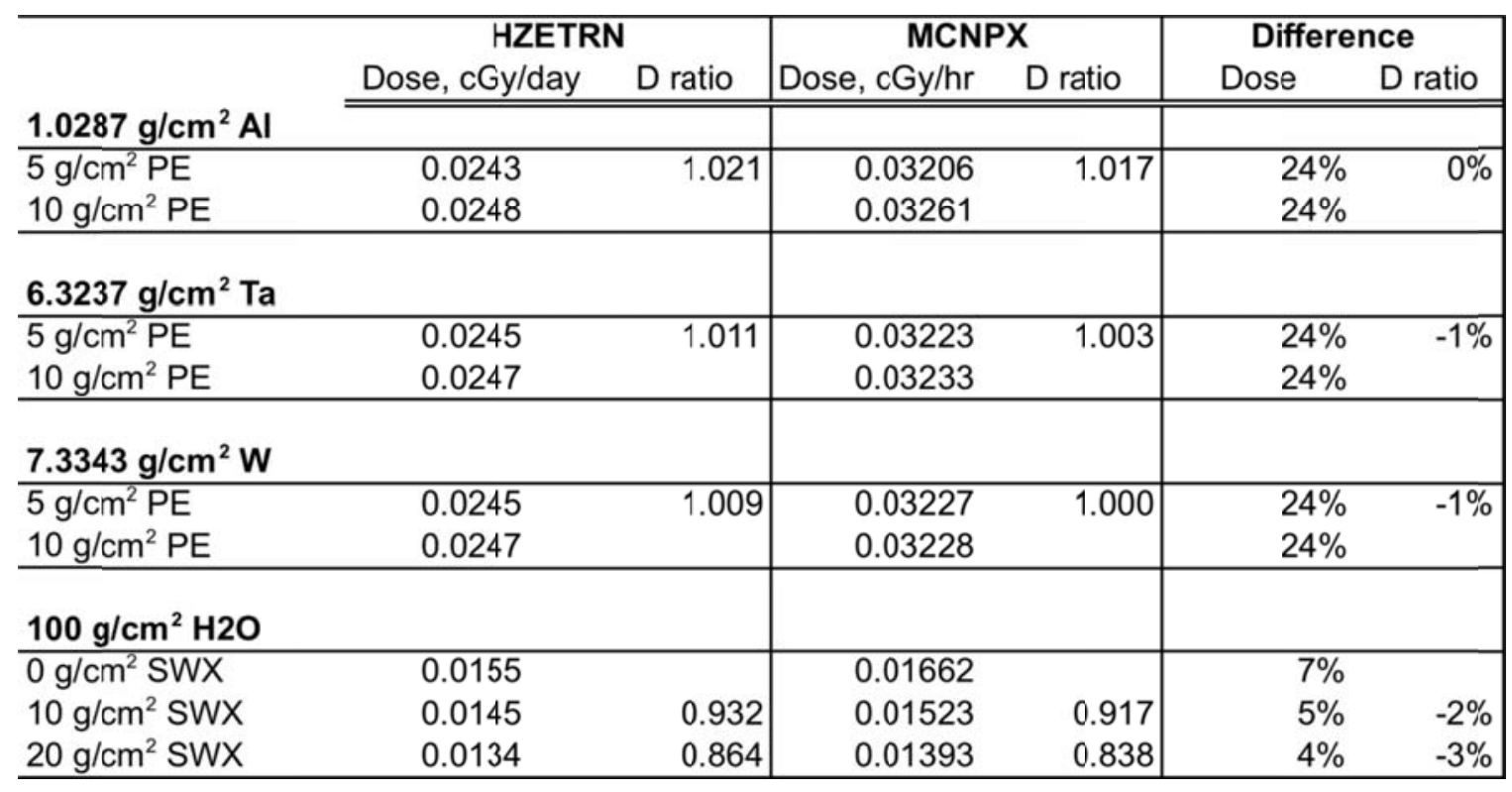

10

American Institute of Aeronautics and Astronautics 
Table 3. Dose values for 1982 GCR (solar maximum).

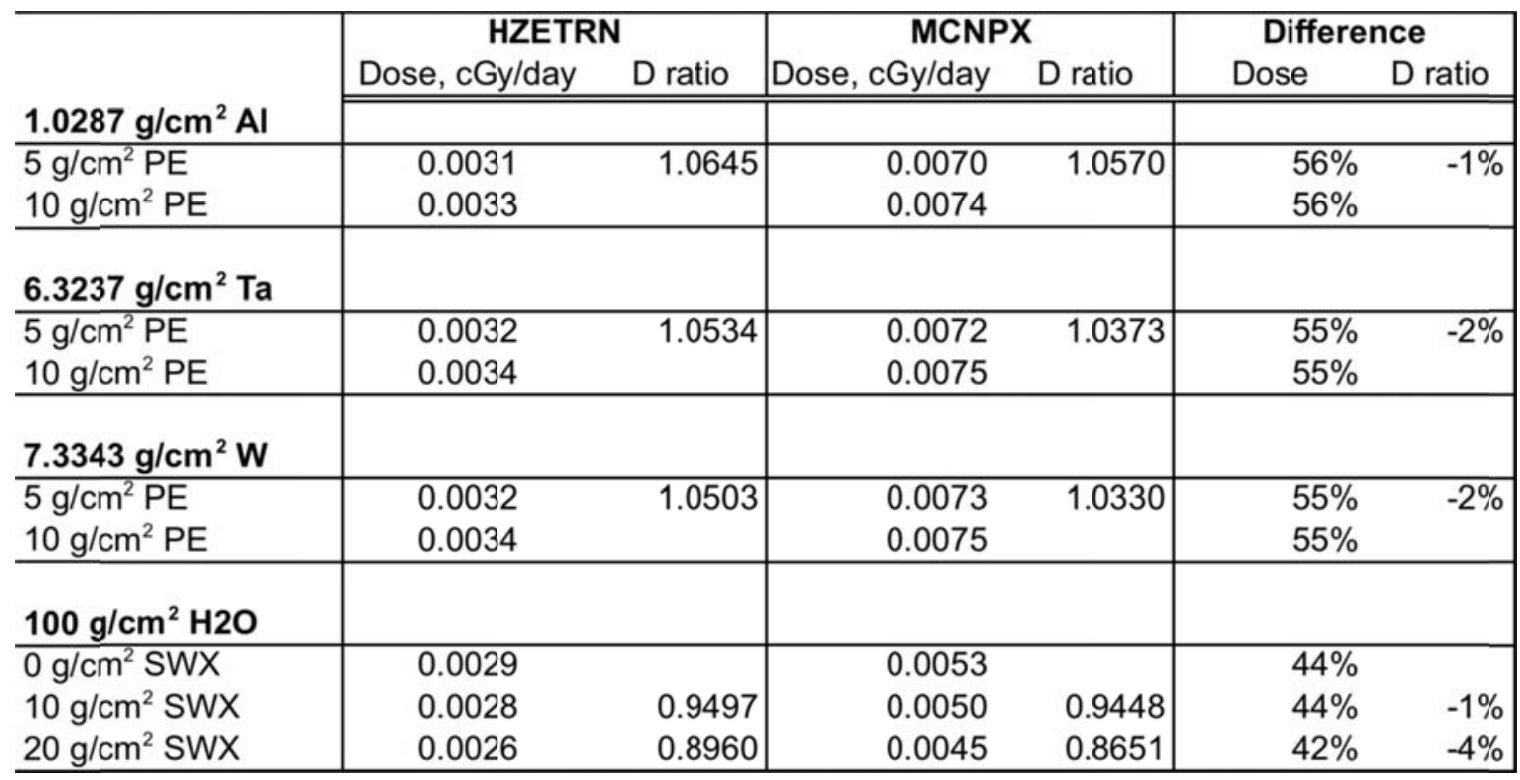

\section{Conclusions}

This study investigated the use of Graded- $Z$ materials for radiation mitigation of proton environments. The results showed some slight dose reductions in using Tantalum or Tungsten with $5 \mathrm{~g} / \mathrm{cm}^{2}$ of HDPE in a GCR environment. However, the Graded-Z configuration was actually detrimental with an SPE environment when compared with a single layer of HDPE. In the very large thickness configurations, the results were comparable.

The verification and validation showed similar trends between HZETRN and MCNPX. The differences in values between the codes were due to known discrepancies in methods of calculation. Further investigation will be needed to elucidate why there are larger differences for the GCR solar minimum environment case.

Furthermore, since this study has only focused on the dose, additional work needs to be implemented to understand whether the incident radiation (protons) or the production of secondary radiation (neutrons) is responsible and how this will affect the dose equivalent. Furthermore, an analysis of the mass of these configurations is important because if we can acquire comparable radiation mitigation characteristics with a Graded$\mathrm{Z}$ configuration, but decrease the overall mass of the system, there is still value in pursuing these Graded- $\mathrm{Z}$ structures. In addition, future work will also investigate three-layer systems to evaluate whether they provide advantages over two-layer or single-layer systems.

\section{References}

${ }^{1}$ Bartholet, B., Atwell, W., Clowdsley, M., Reddell, B., and Wilson, J., “Optimized Spacecraft Material Composition in a HighEnergy Space Radiation Environment,” SPACE-2004, San Diego, CA, Sept. 28-30, 2004.

${ }^{2}$ Atwell, W., Boeder, P., Wilkins, R., and Rojdev, K., "Analyses of Several Space Radiation-Mitigating Materials: Computational and Experimental Results,” SAE International Conference on Environmental Systems (ICES), Savannah, GA, 1216 July 2009.

${ }^{3}$ Fan, W.C., et al., "Shielding Considerations for Satellite Microelectronics", IEEE Transactions on Nuclear Science, Vol. 43, No. 6, 1996, pp. 2790-2796.

${ }^{4}$ Smith, D.M., et al., “The RHESSI Spectrometer,” Solar Physics, Vol. 210, No. 1-2, 2002, pp. 33-60.

${ }^{5}$ Pia, M. G., et al., "PIXE Simulation with Geant4," IEEE Transactions on Nuclear Science, Vol. 56, No. 6, 2009, pp. 36143649.

${ }^{6}$ Fan, W. C., Drumm, C. R., Roeske, S. B., and Scrivner, G. J., “Shielding Considerations for Satellite Microelectronics," IEEE Transactions in Nuclear Science, Vol. 43, No. 6, 1996.

${ }^{7}$ Wilson, J. W., Badavi, F. F., Cucinotta, F. A., Shinn, J. L., Badhwar, G. D., Silberberg, R., Tsao, C.H., Townsend, L.W., and Tripathi, R.K., "HZETRN: Description of a Free-Space Ion and Nucleon Transport and Shielding Computer Program," NASA Technical Paper 3495, 1995.

${ }^{8} \mathrm{MCNPX}$, http://en.wikipedia.org/wiki/MCNPX 
${ }^{9}$ Atwell, W., Tylka, A., Dietrich, W., and Badavi, F. F., "Radiation Exposure Estimates For Extremely Large Solar Proton Events,” 37th Committee on Space Research (COSPAR) Scientific Assembly, Montreal, Canada, 13-20 July 2008.

${ }^{10}$ Allan J. Tylka, William F. Dietrich and William Atwell, "Band Function Representations of Solar Proton Spectra in Ground-Level Events," 38th Scientific Assembly of the Committee on Space Research (COSPAR , Bremen, Germany, 18-25 July 2010.

${ }^{11}$ http://mcnp.lanl.gov/

${ }^{12}$ Aghara, S. K., Blattnig, S. R., Norbury, J. W., and Singleterry, R. C., "Monte Carlo Analysis of Pion Contribution to Absorbed Dose from Galactic Cosmic Rays," Nuclear Instruments and Methods in Physics Research, Section B: Beam Interactions with Materials and Atoms, Vol. 267, No. 7, 2009, pp. 1115-1124.

${ }^{13}$ Slaba, T. C., Blattnig, S. R., Reddell, B., Bahadori, A., Norman, R. B., and Badavi, F. F., "Pion and Electromagnetic Contribution to Dose: Comparisons of HZETRN to Monte Carlo Results and ISS Data," Advances in Space Research, Vol. 52, No. 1, 2013, pp. 62-78.

${ }^{14}$ Slaba, T. C., Blattnig, S. R., Aghara, S. K., Townsend, L. W., Handler, T., Gabriel, T. A., et al., "Coupled Neutron Transport for HZETRN,” Radiation Measurements, Vol. 45, No. 2, 2010, pp. 173-182. 